\title{
Arrest location, initial rhythm and outcome in intensive care unit-treated cardiac arrest patients
}

\section{Purpose of the study}

To evaluate the effect of arrest location and initial rhythm on outcome of intensive care unit (ICU) treated cardiac arrest (CA) patients.

\section{Materials and methods}

A registry-based study to determine associations between CA location and initial rhythm with one-year mortality and neurological outcome in adult CA patients, who received post-arrest treatment in the ICUs of a tertiary hospital between 2005 and 2013.

\section{Results}

Total of 1,139 CA patients

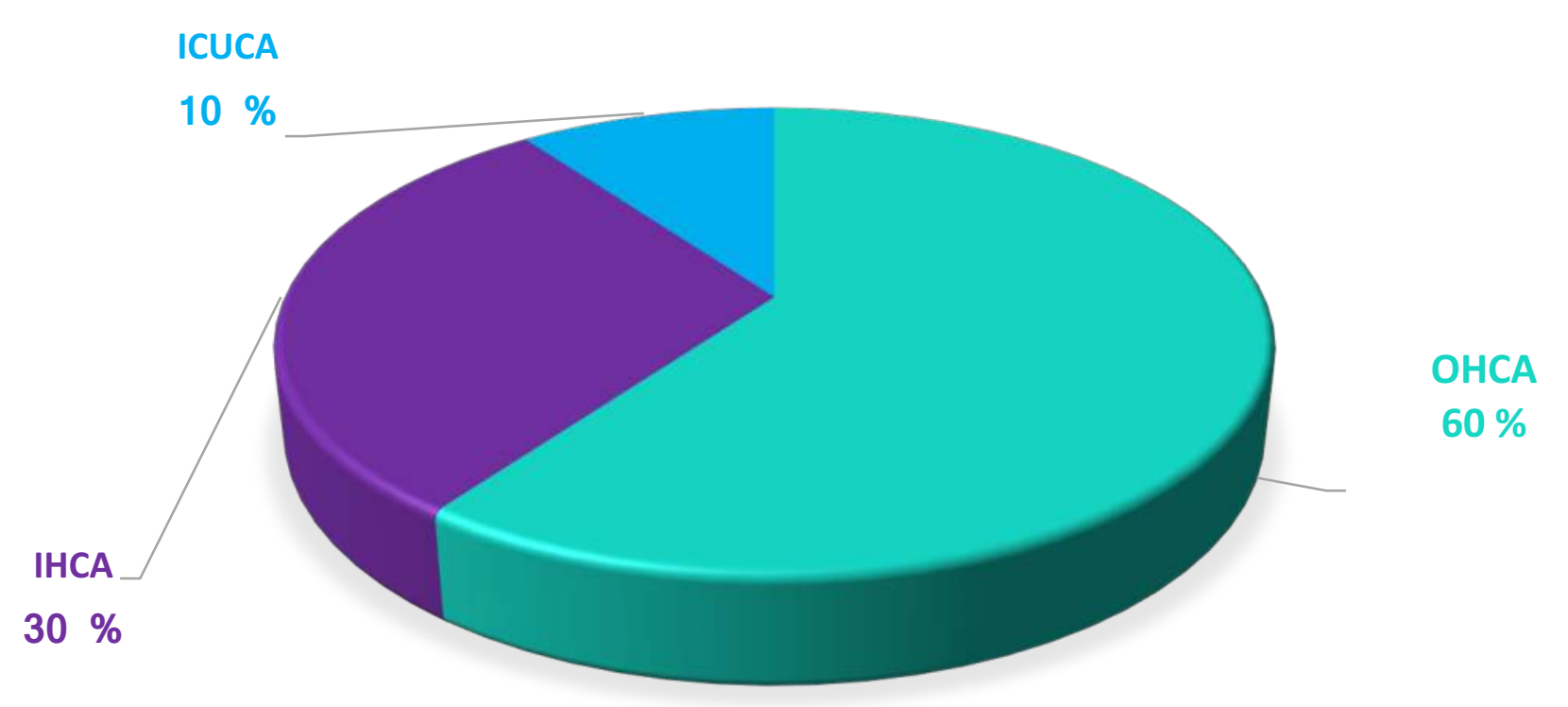

\begin{tabular}{|l|l|l|}
\hline Variable & $\begin{array}{l}\text { Adjusted } \\
\text { Odds Ratio }\end{array}$ & $\begin{array}{l}95 \% \text { confidence } \\
\text { interval }\end{array}$ \\
\hline Age $^{\mathrm{a}}$ & 1.03 & $1.02-1.04$ \\
\hline OHCA & reference & \\
\hline IHCA & 1.45 & $0.97-2.17$ \\
\hline ICUCA & 2.25 & $1.23-4.12$ \\
\hline ROSC & 1.07 & $1.05-1.09$ \\
\hline VF/VT & reference & \\
\hline PEA & 2.07 & $1.4-3.05$ \\
\hline ASY & 3.26 & $1.94-5.46$ \\
\hline
\end{tabular}

${ }^{a}$ per year; ber minute

Initial rhythm: VF/VT 58\%, PEA 25\%, asystole $11 \%$, other/unknown $6 \%$.

Unadjusted one-year mortality was 62\% for in-ICU cardiac arrest patients(ICUCA), $57 \%$ for other in-hospital cardiac arrest patients (IHCA) and $42 \%$ for out-of-hospital cardiac arrest patients (OHCA).
Among one-year survivors, good neurological outcome was seen in $94 \%$ of patients with no significant differences between CA locations or initial rhythms ( $p>0.05$ for both).

\section{Conclusion}

In our study population, OHCA as location and VF/VT as initial rhythm were associated with better survival. Most one-year survivors had a good neurological outcome.
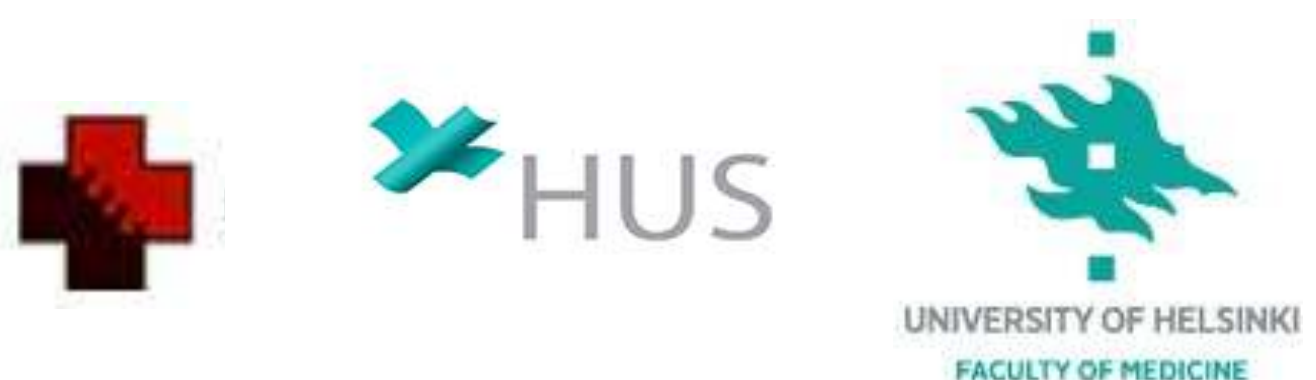

\section{Authors}

I. Efendijev ${ }^{1 *}$, D. Folger ${ }^{1}$, R. Raj $^{1}$, M. Reinikainen ${ }^{2}, M B$. Skrifvars ${ }^{1}$

${ }^{1}$ University of Helsinki and Helsinki University Hospital, Helsinki, Finland

${ }^{2}$ North Karelia Central Hospital, Joensuu, Finland

*ilmar.efendijev@helsinki.fi 